\title{
Europe's Graphene Flagship produces intended results
}

http://graphene-flagship.eu

$\mathrm{L}$ aunched in October 2013 as one of the EU's flagship technology programs with a billion Euros in funding to be spent over a decade, the Graphene Flagship is the largest joint research initiative in Europe today. Its founding aims were to stimulate industrial applications and help boost economies. According to those involved, it is delivering everything that it promised so far.

The Graphene Flagship began with 75 partners from across academia and industry. Funding increases as the project progresses, which means that just $€ 54$ million were received in the first 30 months. In summer 2014, the flagship expanded to welcome 66 new partners, including those from Hungary, Estonia, and Israel. "Now about one-third of the flagship consortium partners are industrial, while at the start they accounted for about one-sixth of the partnership," says Jari Kinaret of Chalmers University of Technology in Sweden, who heads the flagship.

Since 2014, it has also introduced more than 25 associate members from industry, who are not funded by the flagship but by other means. They include the ABB Group, the multinational manufacturer of automation and process control systems, and Germany-based Netzsch Group, which manufactures machinery and instrumentation. According to Andrea Ferrari, Director of the Cambridge Graphene Centre and the Graphene Flagship's Science and Technology

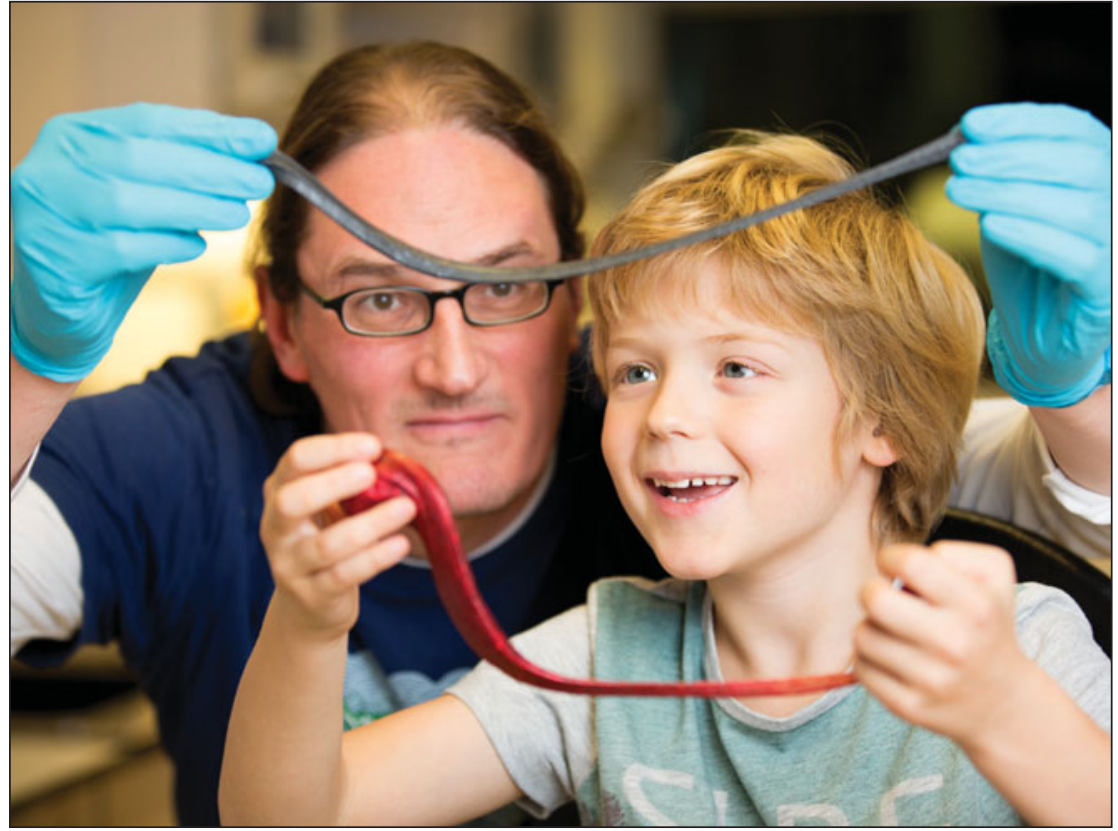

Jonathan Coleman at Trinity College Dublin's School of Physics examines a piece of "g-putty," while his son, Oisín, plays with Silly Putty. Photo by Naoise Culhane; courtesy of AMBER, Trinity College Dublin.
Officer, there are plans to accept more associate members in the future.

"I believe that the flagship at the moment is going well," Ferrari says. Kinaret adds, "We are well on our way, and have met or exceeded all our targets, sometimes by an embarrassing margin. For instance, we expected to publish 300 publications in the first 30 months and did publish almost 800 ."

One success story was published in December 2016, after researchers from The University of Manchester in the UK and Trinity College Dublin found that adding a small amount of graphene to children's Silly Putty could transform it into a highly sensitive pressure sensor. The resulting gray goo, dubbed "g-putty," is not only able to conduct electricity but its electrical resistance varies with even the slightest pressure, such as that of a heartbeat. Around $40 \%$ of the funding for this research came from the flagship.

According to physicist professor Jonathan Coleman at Trinity College Dublin, who worked on g-putty, the benefit of being in the flagship is not purely financial. "The flagship is a really great vehicle to enable cooperation," he says.

Another success is at the University of Trieste, Italy, where work has been done on interfacing graphene with neurons in the hope of developing better brain implants. And at the University of Cambridge, researchers are working on graphene-based rechargeable batteries with a $20 \%$ higher energy efficiency than lithium-ion batteries.

At its launch, Kinaret told $M R S$ Bulletin (May 2014, p. 393) that he hoped the Graphene Flagship would stimulate applied innovation. Some small progress has been seen on this front. In Spain, the automotive interiors company Grupo 
Antolin developed a kayak that incorporates graphene into its thermoset polymeric matrices, improving strength and stiffness. The Italian Institute of Technology and luxury Italian design brand Momodesign together produced a motorcycle helmet with a graphene coating, allowing for better distribution of force during impact even under hightemperature conditions.

So far, partners in the flagship have released around 10-15 new products, most of which are different types of graphene materials. "The project has only been running less than [a] third of its allotted time and used about one-sixth of its budget so it would be rather surprising if we had reached all our goals already," Kinaret says. The aim for the next phase of the flagship is to focus more heavily on boosting industrial impact. "In some areas, such as supercapacitors, we are already approaching the pilot line stage, while in other fields we expect to produce system-level demonstrators," he says.

One more challenge is how the Graphene Flagship will cope with the departure of the UK from the EU following the decision made in a referendum by the British public in June 2016 known as "Brexit." The UK has always been a key player in the flagship, not least because the discovery of graphene itself was made by a Nobel Prize-winning team at The University of Manchester. Currently, 17 of the partner organizations are in the UK. According to Ferrari, the flagship's official position for now is "business as usual." He says that since no one is yet sure what Brexit will actually mean when it happens, "It's very difficult to speculate."

Angela Saini
NIST studies recommend closing tech gaps to fortify advanced manufacturing

$\mathrm{T}_{\mathrm{s}}^{\mathrm{o}}$ o spur significant innovation and growth in advanced manufacturing, as well as save over $\$ 100$ billion annually, US industry must rectify currently unmet needs for measurement science and "proof-of-concept" demonstrations of emerging technologies. This is the overall conclusion reached by economic studies funded by the National Institute of Standards and Technology (NIST) of four advanced manufacturing areas used to create everything from automobile composites to zero-noise headsets.

"Gaps in the technology infrastructure - including the lack of reliable measurement and test methods, scientifically based standards, and other formal knowledge and tools - limit advanced manufacturing's further development and adoption," says NIST economist Gary Anderson, coordinator of the economic studies prepared by RTI International, an independent nonprofit research institute.

Using data collected through extensive interviews and surveys with researchers, developers, manufacturers, and other stakeholders, each of the four studies identifies 5-10 critical technical barriers to the adoption of its specific manufacturing technology. The studies also estimate the impacts of eliminating these obstacles and define which needs should be met first to do so.
For example, establishing industrywide standards and measurements for the inks and substrates used in roll-to-roll (R2R) manufacturing - the fabrication of electronic devices on a roll of flexible plastic or metal - is projected to reduce production costs by $15 \%$. Likewise, the development and adoption of verified reference data, robust measurement technologies and testing protocols, and standardized modeling and finishing methods could yield some $\$ 4$ billion in annual benefits and savings for additive manufacturing, a process also known as three-dimensional printing.

The two largest predicted cost savings were the $\$ 57.4$ billion and $\$ 40.1$ billion for smart manufacturing (where all manufacturing data from design to finished product is electronically exchanged and processed) and advanced robotics and automation sectors, respectively. Among the needs that must be met to realize both of these benefits, the researchers say, is increasing access by small- and mediumsized manufacturers to the same state-ofthe-art methods, tools, and knowledge as their larger counterparts.

For each of the four advanced manufacturing technologies studied, the estimated annual cost savings and percentage reduction in production costs are:

- Additive manufacturing: \$4.1 billion, $18.3 \%$

\section{- Advanced robotics and automation:} \$40.1 billion, 5.3\%

- R2R manufacturing: \$400 million, $14.7 \%$

- Smart manufacturing: $\$ 57.4$ billion, $3.2 \%$

The researchers state that their studies only looked at benefits directly attributable to closing the identified technical gaps in each sector; therefore, the impact estimates are conservative. "If we consider the larger-scale outcomes brought about by meeting these needs - such as new and improved products, increased production quality, long-term industry growth and job creation - the impacts would be significantly higher," Anderson says.

The studies also support a number of key strategies for overcoming technical barriers and fortifying advanced manufacturing, including:

- keeping standards and performance measures nonproprietary,

- using public research institutions to develop those tools, and

- working through manufacturing research consortia and technology extension services to ensure that all manufacturers - especially small- and medium-sized enterprises-can access them.

"Our studies emphasize that full economic impact will only be realized if all technical needs are met, and all stakeholders regardless of size, not just large manufacturers, can share in the rewards," Anderson says. 